\title{
Apelin Ameliorates High Glucose-Induced Downregulation of Connexin 43 via AMPK-Dependent Pathway in Neonatal Rat Cardiomyocytes
}

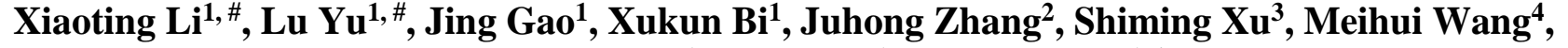 \\ Mengmeng Chen', Fuyu Qiu', Guosheng Fu' ${ }^{1}$ ** \\ ${ }^{1}$ Department of Cardiology, Sir Run Run Shaw Hospital, Zhejiang University School of Medicine, Hangzhou, \\ Zhejiang, China \\ ${ }^{2}$ Zhejiang University School of Medicine, Hangzhou, Zhejiang, China \\ ${ }^{3}$ Institute of Translational Medicine, Zhejiang University School of Medicine, Hangzhou, Zhejiang, China \\ ${ }^{4}$ Biomedical Research Center, Sir Run Run Shaw Hospital, Zhejiang University School of Medicine, Hangzhou, \\ Zhejiang, China
}

[Received January 27, 2017; Revised April 24, 2017; Accepted April 26, 2017]

\begin{abstract}
Diabetes Mellitus is a common disorder, with increasing risk of cardiac arrhythmias. Studies have shown that altered connexin expression and gap junction remodeling under hyperglycemia contribute to the high prevalence of cardiac arrhythmias and even sudden death. Connexin 43 (Cx43), a major protein that assembles to form cardiac gap junctions, has been found to be downregulated under high glucose conditions, along with inhibition of gap junctional intercellular communication (GJIC). While, apelin, a beneficial adipokine, increases $\mathrm{Cx} 43$ protein expression in mouse and human embryonic stem cells during cardiac differentiation. However, it remains unknown whether apelin influences GJIC capacity in cardiomyocytes. Here, using Western blotting and dye transfer assays, we found that $\mathrm{Cx} 43$ protein expression was reduced and GJIC was impaired after treatment with high glucose, which, however, could be abrogated after apelin treatment for $48 \mathrm{~h}$. We also found that apelin increased Cx43 expression under normal glucose. Real-time PCR showed that the $\mathrm{Cx43}$ mRNA was not significantly affected under high glucose conditions in the presence of apelin or high glucose and apelin. High glucose decreased the phosphorylation of AMPKa; however, apelin activated AMPKa. Interestingly, we found that $\mathrm{Cx} 43$ expression was increased after treatment with AICAR, an activator of AMPK signaling. AMPKa inhibition mediated with transfection of siRNA-AMPKa1 and siRNA-AMPKo2 abolished the protective effect of apelin on Cx43 expression. Our data suggest that apelin attenuates high glucose-induced $\mathrm{Cx} 43$ downregulation and improves the loss of functional gap junctions partly through the AMPK pathway.
\end{abstract}

Key words: cardiomyocytes, connexin 43, high glucose, apelin, gap junction, AMPK

Diabetic cardiac dysfunction is characterized by mechanical and electrical abnormalities, which, in turn, result in a high prevalence of cardiac arrhythmias and sudden death [1]. Altered connexin expression and gap junction remodeling contribute to the high susceptibility of diabetic hearts to arrhythmias [2, 3]. Connexin 43
(Cx43) is the most extensive expressed connexin that assembles to form gap junctions in mammalian ventricular muscle [4-6]. Abnormal $\mathrm{Cx} 43$ expression and location were found in hypertrophic, ischemic and diabetic hearts [7-10]. Accordingly, hyperglycemia is a pivotal initiator for diabetic cardiac complications [11],

*Correspondence should be addressed to: Dr. Guosheng Fu, Sir Run Run Shaw Hospital, Zhejiang University School of Medicine, Hangzhou, 310016, Zhejiang, China. Email: fu guosheng@163.com. ${ }^{\#}$ These authors contributed equally to this work.

Copyright: () $2017 \mathrm{Li} \mathrm{X}$ et al. This is an open-access article distributed under the terms of the Creative Commons Attribution License, which permits unrestricted use, distribution, and reproduction in any medium, provided the original author and source are credited. 
and previous studies have reported that gap junctional intercellular communications (GJICs) are impaired and $\mathrm{Cx} 43$ expression is reduced in neonatal rat cardiomyocytes (NRCMs) exposed to high glucose [12, 13].

Apelin is a beneficial adipokine that is widely present in numerous tissues, such as the heart, lung, kidney, liver, and adipose tissue. Tatemoto, et al. firstly found the endogenous ligand named apelin for Angiotensin II receptor-like 1 (APJ) from bovine stomach homogenates in 1998 [14]. Apelin gene encodes for a 77amino acid pre-propeptide, and prepro-apelin can be cleaved into different bioactive apelin fragments, including apelin-36, apelin-17 and apelin-13 [15]. Among them, apelin-13 is predominant in the heart [16, 17]. Apelin/APJ system has been considered to play a key role in various physiological processes, such as energy metabolism, angiogenesis, cardiovascular functions and fluid homeostasis [17, 18]. Experimental evidence from various studies has indicated that apelin potently improves cardiac contractility [19-21] and alleviates ischemia-reperfusion injury $[22,23]$. Generally, the cardioprotective effect of apelin in diabetic hearts is that apelin stimulates neovascularization, improves glucose uptake and insulin sensitivity in cardiomyocytes [24]. Several studies observed low plasma levels of apelin in patients with atrial fibrillation [25-27]. During cardiac differentiation of mouse and human embryonic stem cells, the increased expression of $\mathrm{Cx} 43$ was found in group treated with apelin [28]. However, it remains unclear whether apelin can treat arrhythmias.

Adenosine monophosphate-activated protein kinase (AMPK) is a serine-threonine kinase composed with three different subunits: a catalytic subunit $(\alpha)$, a scaffolding subunit $(\beta)$ and a regulatory subunit $(\gamma)$. AMPK regulates energy metabolism and many other cellular processes involved in overall cell health, such as cell growth, autophagy, apoptosis, and regulation of cardiac sodium and potassium channels [29-31]. AMPK also plays an important adaptive role in a variety of arrhythmiapromoting cardiovascular diseases and can modify arrhythmogenic conditions [32]. Moreover, several studies have demonstrated that $\mathrm{Cx} 43$ expression was regulated by AMPK activity [29, 33, 34].

Even though apelin has been found to increases $\mathrm{Cx} 43$ expression in embryonic stem cells, whether apelin improves GJIC and the underlying mechanisms remain unknown. In this study, we aimed to identify the effects of apelin on the expression and function of gap junctions in NRCMs under high glucose treatment and to investigate whether AMPK was implicated in this condition.

\section{MATERIALS AND METHODS}

\section{Chemicals and Reagents}

Apelin-13, glucose, mannitol, Lucifer Yellow $\mathrm{CH}$ dilithium salt, and Rhodamine-dextran were purchased from Sigma (St. Louis, USA). AICAR was purchased from Selleck (Houston, USA). Dulbecco's modified Eagle's medium(DMEM) and Opti-MEM (reduced serum media) were obtained from GIBCO (Grand Island, NY, USA). Newborn calf serum (NBCS) was supplied by Tianhang Biotechnology (Hangzhou, China). Polyclonal rabbit antibodies including anti-Cx43 antibody, antiAMPK $\alpha$, and anti-phospho-AMPK $\alpha$, were obtained from Cell Signaling Technology (Massachusetts, USA). $\beta$ tubulin monoclonal antibody (HRP conjugated) was purchased from MultiSciences (Hangzhou, China).

\section{Cell Culture and Treatment}

NRCMs were prepared from 1-to-2-day-old SpragueDawley rats (provided by the Experimental Animal Center of Zhejiang Province), and the animal experiments were approved by the Institutional Animal Care and Use Committee of Zhejiang University. NRCMs were isolated and cultured as previously described [12]. Breifly, hearts were dissected and digested in $0.12 \%$ trypsin and $0.1 \%$ collagenase II at $37^{\circ} \mathrm{C}$. The cell suspension was then cultured in DMEM (5.5 mM glucose) with $10 \%(\mathrm{v} / \mathrm{v})$ NBCS in a humidified $37^{\circ} \mathrm{C}, 5 \% \mathrm{CO}_{2}$ incubator (Thermo, MA, USA). The fibroblasts were deducted from the cell suspension by pre-plating for $90 \mathrm{~min}$ on account of differential cell adhesion. Cells were seeded $1 \times 10^{6} /$ well in 6-well plates. Cell culture medium was replaced every 48 h. Apelin-13 was dissolved in sterilized phosphate buffer solution (PBS, pH 7.4) and used for treating cells in various concentrations as indicated. Mannitol $(24.5 \mathrm{mM})$ was dissolved in DMEM (5.5 mM glucose) as an osmotic control.

\section{Western Blotting Analysis}

Cells in 6-well plates were extracted in a radioimmunoprecipitation assay (RIPA) lysis buffer containing $1 \mathrm{mM}$ phenylmethylsulfonyl fluoride (PMSF). Proteins were quantified with a bicinchoninic acid (BCA) assay kit (Beyotime Biotechnology, Shanghai, China). Samples containing equal amounts of protein were separated using $10 \%$ SDS polyacrylamide gels, transferred onto polyvinylidene difluoride membranes (PVDF, Bio-Rad, CA, USA), and blocked for $1 \mathrm{~h}$ in Trisbuffered saline and $0.1 \%$ Tween 20 (TBST) with 5\% (w/v) nonfat dry milk at room temperature. Next, PVDF membranes were incubated with primary antibodies 
overnight at $4^{\circ} \mathrm{C}$ followed by HRP-linked secondary antibodies for $1 \mathrm{~h}$ at room temperature after 3 TBST washes. The bands were exposed by reacting with enhanced chemiluminescence (ECL) reagents on an

Amersham Imager 600 system (GE Healthcare, Buckinghamshire, England).

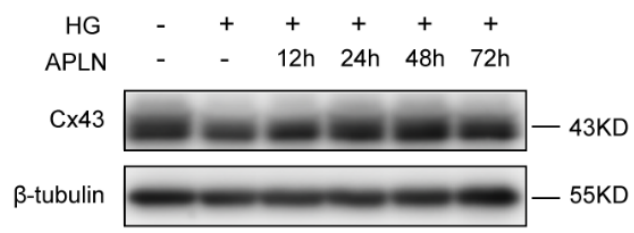

B
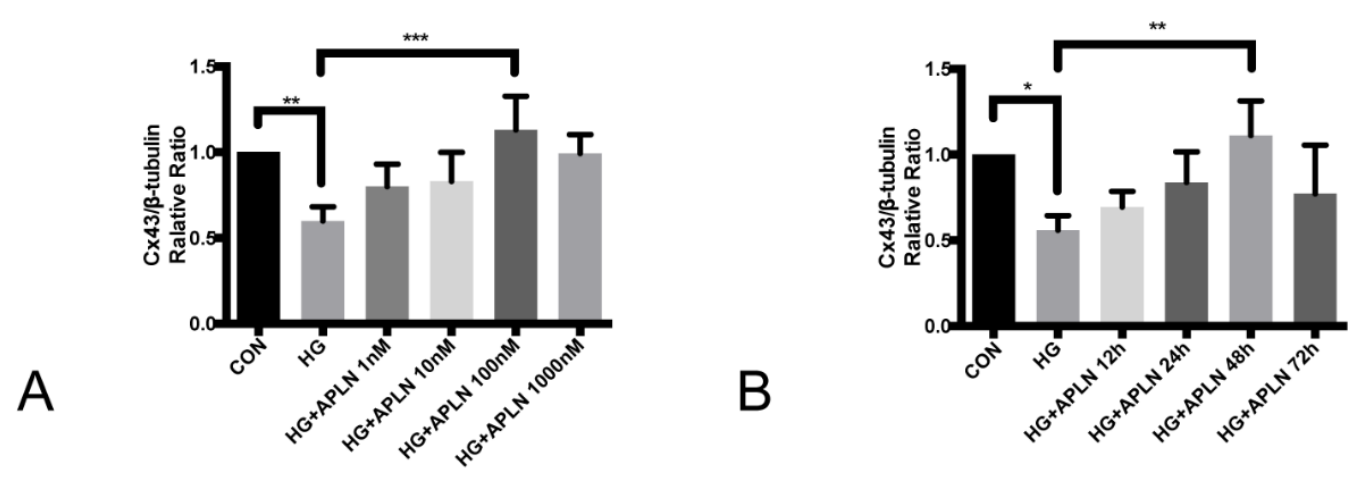

A
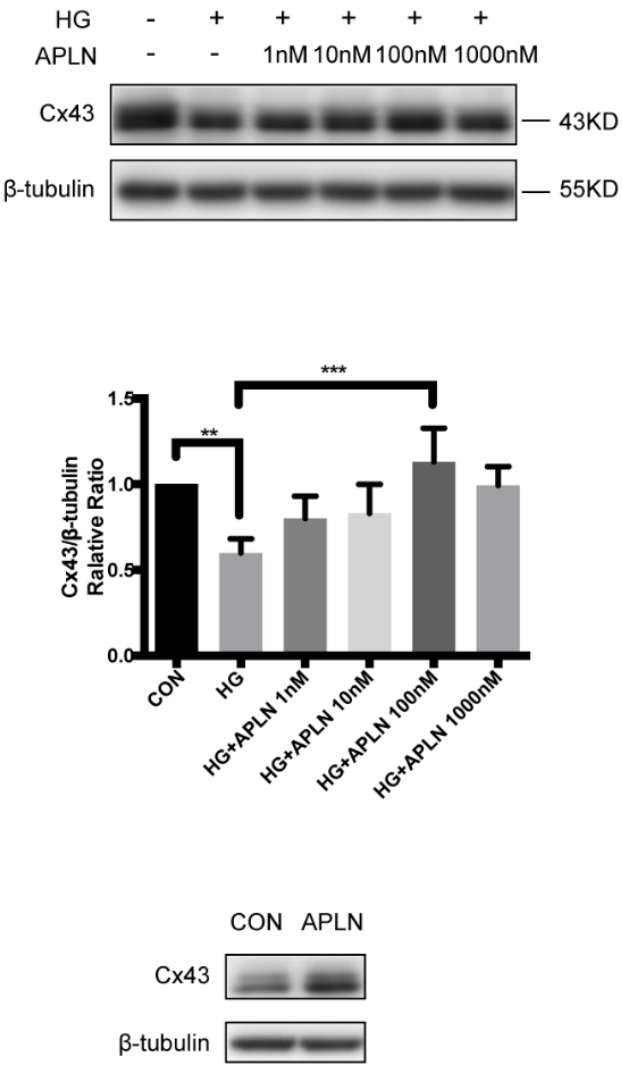

C

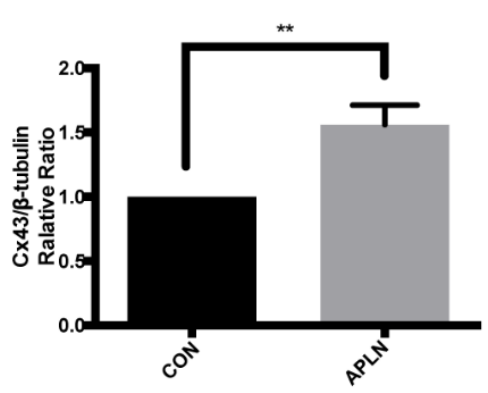

Figure 1. Effects of apelin-13 on Cx43 expression in NRCMs. Apelin-13 reversed the Cx43 downregulation triggered by high glucose in a concentration- (A) and time-dependent (B) manner. The expression of Cx43 significantly decreased under high glucose condition (30 mM, $72 \mathrm{~h})$. The addition of apelin-13 (100 nM, $48 \mathrm{~h}$ ) apparently alleviated the Cx43 reduction induced by high glucose. (C) Incubation with $100 \mathrm{nM}$ apelin-13 only for $48 \mathrm{~h}$ increased the Cx43 protein expression. (D) The effects of high glucose and/or apelin-13 on Cx43 mRNA level were quantified by real-time PCR. CON: normal glucose $(5.5 \mathrm{mM})$; HG: high glucose $(30 \mathrm{mM})$; APLN: apelin-13 (100 nM). $* P<0.05, * * P<0.01$, $* * * P<0.001$. Data are the mean $\pm \mathrm{SD}$. 


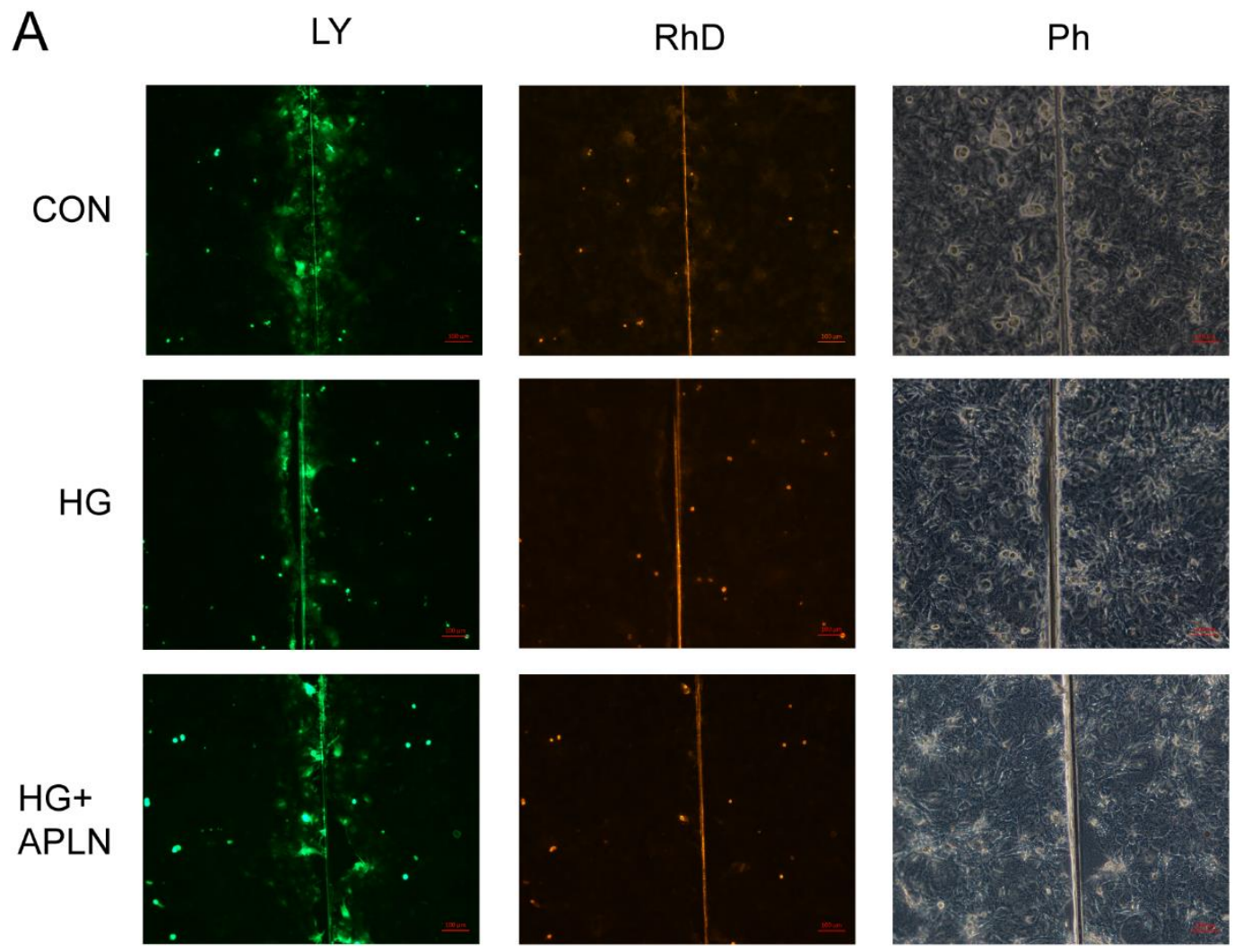

$\mathrm{B}$

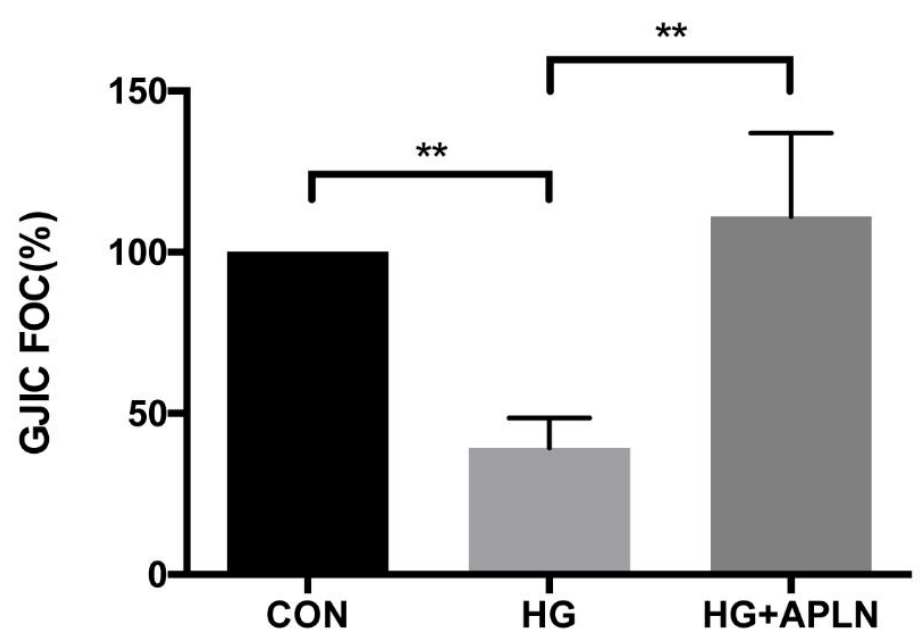

Figure 2. Effects of apelin-13 on high-glucose induced GJIC in NRCMs. The GJIC capacity of NRCMs was evaluated by dye transfer assay. (A) Cells were left untreated in the control group. (B) Cells were incubated with 30 $\mathrm{mM}$ glucose for $72 \mathrm{~h}$. (C) Cells were initially treated with $30 \mathrm{mM}$ glucose for $24 \mathrm{~h}$ and then co-treated with $100 \mathrm{nM}$ apelin-13 for the following $48 \mathrm{~h}$. (D) The statistical data of net LY dye transfer area indicated that high glucose significantly reduced the LY transfer area and that apelin-13 rescued the high glucose-induce transfer area to a normal level. CON: normal glucose (5.5 mM); HG: high glucose (30 mM); APLN: apelin-13 (100 nM). $* P<0.05, * * P<0.01$, $* * * P<0.001$. Data are the mean $\pm \mathrm{SD}$. 


\section{qRT-PCR}

Total RNA was extracted with TRIZOL reagent (CWBIO, Beijing, China) and converted into cDNA using a PrimeScript RT reagent Kit (Takara, Tokyo, Japan) according to the manufacturer's instructions. Real-time PCR was performed on the Viia 7 system (Applied Biosystems, CA, USA) using UltraSYBR Mixture (Low ROX) (CWBIO, Beijing, China). The following primers were used: $\mathrm{Cx} 43$ forward, 5'-TCTGCCTTTC GCTGTAACACT-3', reverse, 5'-GGGCACAGACAC GAATATGAT-3'; $\alpha$-tubulin forward, 5'-GACGGA TGCAGAAGGAGATTACT-3', reverse, 5'-TGATCCA CATCTGCTGG AAGGT-3'.

\section{Scrape Loading/Dye Transfer Assay}

The scrape loading/dye transfer (SL/DL) assay was used to assess gap junctional intercellular communication (GJIC). Lucifer yellow (LY, MW 457), a membrane impermeable fluorescent dye that can go across junctional channels, is the most popular dye in research use. Rhodamine-dextran (Rhd, MW 10000), which is too large to transverse the junctional channels, is often used as an additional control to identify the cells initially loaded after the scrape [35]. After treatment with high glucose and apelin, cells seeded in $35 \mathrm{~mm}$ dishes were rinsed gently three times with PBS (pre-warmed to $37^{\circ} \mathrm{C}$ ). Sufficient 1 $\mathrm{mg} / \mathrm{mL} \mathrm{LY}$-dye and $1 \mathrm{mg} / \mathrm{mL}$ rhodamine-dextran mixed solution $\left(37^{\circ} \mathrm{C}\right)$ was added to the dishes, and then the cells were scraped with a surgical steel blade. After incubation at $37^{\circ} \mathrm{C}$ for $4 \mathrm{~min}$, the cells were washed three times with warmed PBS and then fixed by adding $4 \%$ paraformaldehyde before visualization with a fluorescent microscope (Zeiss, Oberkochen, Germany). National Institute of Health (NIH) imaging system was used to calculate the fluorescence area. The fraction of the control (GJIC-FOC) was used to evaluate the GJIC capacity [35]. GJIC FOC $_{\text {Treatment }}=\left(\right.$ Area $_{\text {Treatment }}{ }^{\mathrm{LY}}-$ Area Treatment $\left.^{\text {Rhd }}\right) /$ (AreaControl $^{\mathrm{LY}}$ - AreaControl ${ }^{\mathrm{Rhd}}$ )

\section{Transfection of siRNA}

The siRNAs against AMPK $\alpha 1$ and AMPK $\alpha 2$ were designed and synthesized by Sigma (St. Louis, USA). An unsilencing siRNA was used as a negative control. The siRNA-AMPK $\alpha 1$ and siRNA-AMPK $\alpha 2$ sequence were as follows: siRNA-AMPK $\alpha 1$ sense strand, 5'-CUAUGAAU GGAAGGUUGUAdTdT-3', anti-sense strand, 5'-UAC AACCUUCCAUUCAUAGdTdT-3'; siRNA-AMPK $\alpha 2$ sense strand, 5'-GCUUUACCUGGUUGACAAUdTdT3', anti-sense strand, 5'-AUUGUCAACCAGGUAAAG CdTdT-3'.

The siRNA and Hiperfect transfection reagents (Qiagen, Hilden, Germany) were premixed with OptiMEM at room temperature for $10 \mathrm{~min}$. Cells in 6-well plates were incubated with $500 \mu \mathrm{L}$ of mixed solution containing $100 \mathrm{nM}$ siRNA for $6 \mathrm{~h}$ and then added to 1500 $\mu \mathrm{L}$ of DMEM. High glucose and apelin were added $48 \mathrm{~h}$ after the transfection.
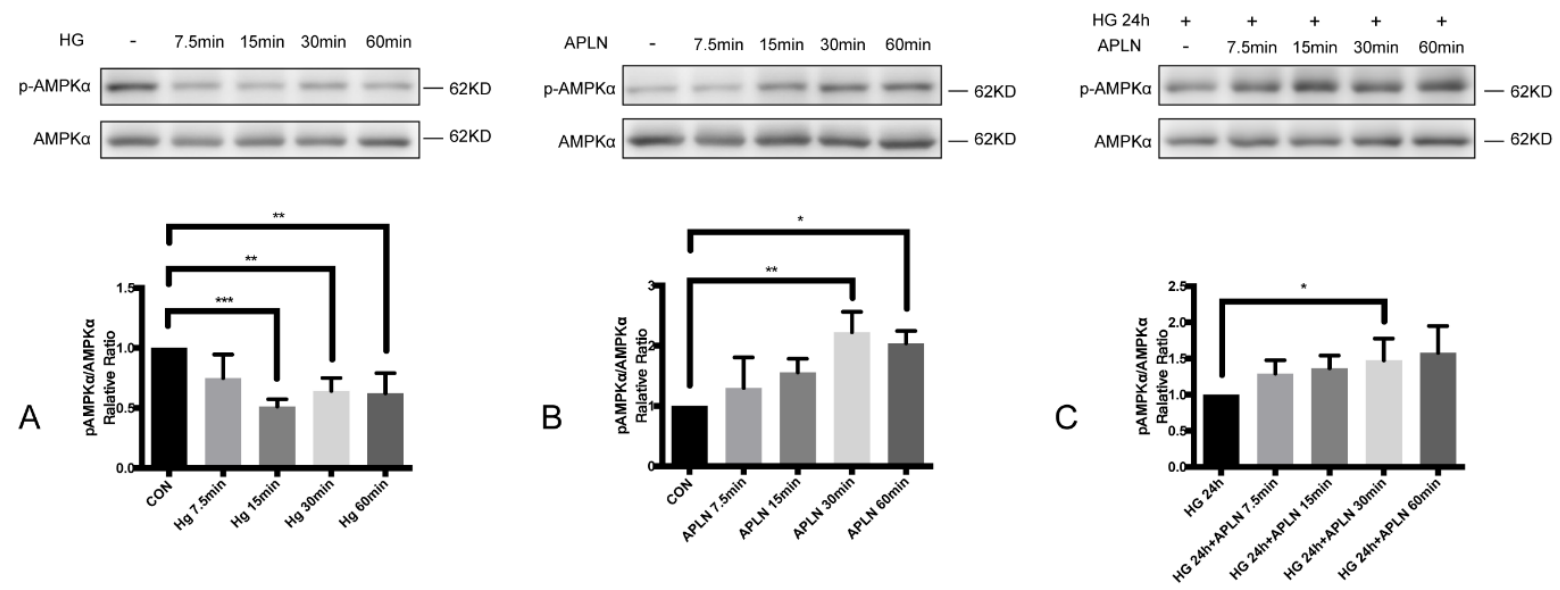

Figure 3. The activity of AMPK induced by high glucose and apelin in NRCMs. (A) Cells were separately treated with 30 $\mathrm{mM}$ glucose for $7.5 \mathrm{~min}, 15 \mathrm{~min}, 30 \mathrm{~min}$ and $60 \mathrm{~min}$. The p-AMPK $\alpha / \mathrm{AMPK} \alpha$ ratio declined significantly after high glucose treatment for $7.5 \mathrm{~min}$. (B) The phosphorylation of AMPK $\alpha$ increased after the administration of apelin-13 for 30min. (C) Cells were pre-treated with $30 \mathrm{mM}$ glucose for $24 \mathrm{~h}$, and the level of pAMPK $\alpha / \mathrm{AMPK} \alpha$ increased at $30 \mathrm{~min}$ after adding apelin. CON: normal glucose (5.5 mM); HG: high glucose (30 mM); APLN: apelin-13 (100 nM). $* P<0.05, * * P<0.01, * * * P<0.001$. Data are the mean \pm SD. 


\section{Statistical Analysis}

Results were expressed as mean $\pm \mathrm{SD}$, and experiments were repeated for at least three times. The statistical analysis was performed using one-way ANOVA. Significant differences were considered at $P<0.05$.

\section{RESULTS}

\section{Apelin-13 attenuated the Cx43 downregulation induced by high glucose in NRCMs.}

Cells were pre-incubated with $30 \mathrm{mM}$ high glucose for 24 $\mathrm{h}$, and then co-treated with apelin-13 at the indicated concentrations $(1 \mathrm{nM}, 10 \mathrm{nM}, 100 \mathrm{nM}, 1000 \mathrm{nM})$ for the following $48 \mathrm{~h}$. Apelin-13 was demonstrated to reverse the $\mathrm{Cx} 43$ protein reduction in a dose-dependent manner with a peak at $100 \mathrm{nM}$ (Fig. 1A). NRCMs were treated with apelin-13 (100 $\mathrm{nM})$ at various times $(12,24,48$, and $72 \mathrm{~h}$ ) under $30 \mathrm{mM}$ high glucose conditions for $72 \mathrm{~h}$. The expression of $\mathrm{Cx} 43$ was reduced under high glucose, and the decrease was mitigated by treatment with apelin- 13 in a time-dependent manner with a peak at $48 \mathrm{~h}$. The protective effect of apelin seemed to evaporate at $72 \mathrm{~h}$
(Fig. 1B). In cells treated with apelin-13 (100 nM, $48 \mathrm{~h}$ ) only, $\mathrm{Cx} 43$ expression was significantly increased as well (Fig. 1C). However, the level of $\mathrm{Cx} 43$ mRNA was not affected by either high glucose or co-treatment with apelin-13 (Fig. 1D).

\section{Apelin-13 improved the high glucose-induced GJIC.}

To examine the influence of apelin-13 on the function of gap junctions, a dye transfer assay was applied to measure the GJIC. In the control group, cells were found to remain well coupled by monitoring the net Lucifer Yellow areas. Under high glucose conditions ( $30 \mathrm{mM}, 72 \mathrm{~h}$ ), the net LY transfer area was significantly reduced. However, the addition of apelin-13 (100 nM, $48 \mathrm{~h}$ ) to cells effectively ameliorated the reduced area caused by high glucose (Fig. 2A). The quantitative data of net LY transfer area indicated that half of the area reduced under high glucose condition was rescued to normal levels following apelin treatment (Fig. 2B). These results indicated that apelin-13 can protect the GJIC capacity from high glucose conditions.

A
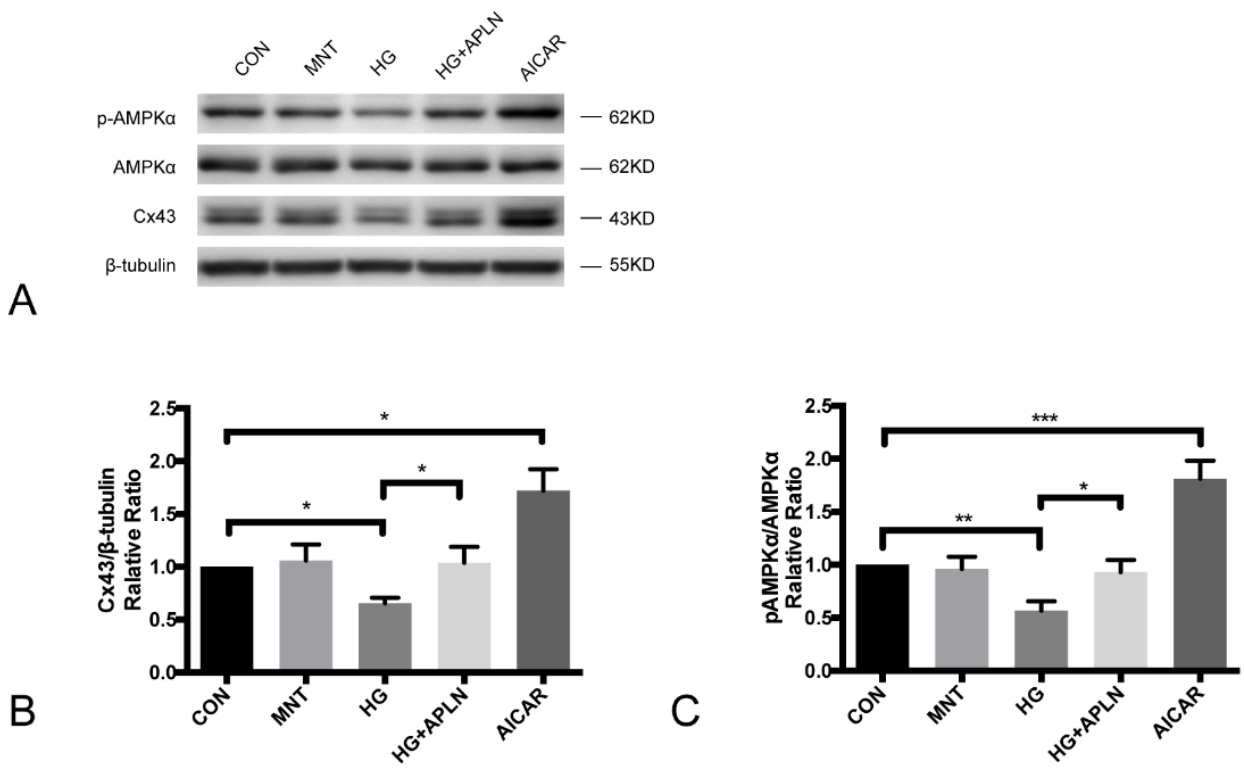

Figure 4. The effect of AICAR on Cx43 and pAMPK expression in NRCMs. (A) Cells incubated under normal glucose (CON), osmotic control (MNT), high glucose (HG), high glucose with apelin (HG+APLN) and AICAR were harvested for western blotting. (B) and (C) Compared with control group, the Cx43 expression and pAMPK/AMPK $\alpha$ ratio in osmotic control group were not significantly different. The expression and of $\mathrm{Cx} 43$ and pAMPK in high glucose group $(30 \mathrm{mM}, 72 \mathrm{~h})$ was significantly reduced compared with control group, and co-treatment with apelin $(100 \mathrm{nM}, 48 \mathrm{~h})$ relieved the downregulated Cx43 and pAMPK expression. Compared with control group, pAMPK $\alpha$ and $\mathrm{Cx} 43$ expression was significantly increased in AICAR group.CON: normal glucose (5.5 mM); MNT: normal glucose (5.5 mM) and mannitol $(24.5 \mathrm{mM})$; HG: high glucose $(30 \mathrm{mM})$; APLN: apelin-13 (100 nM). AICAR: an AMPK activator $(0.5 \mathrm{mM}) . * P<0.05$, $* * P<0.01, * * * P<0.001$. Data are the mean $\pm \mathrm{SD}$. 

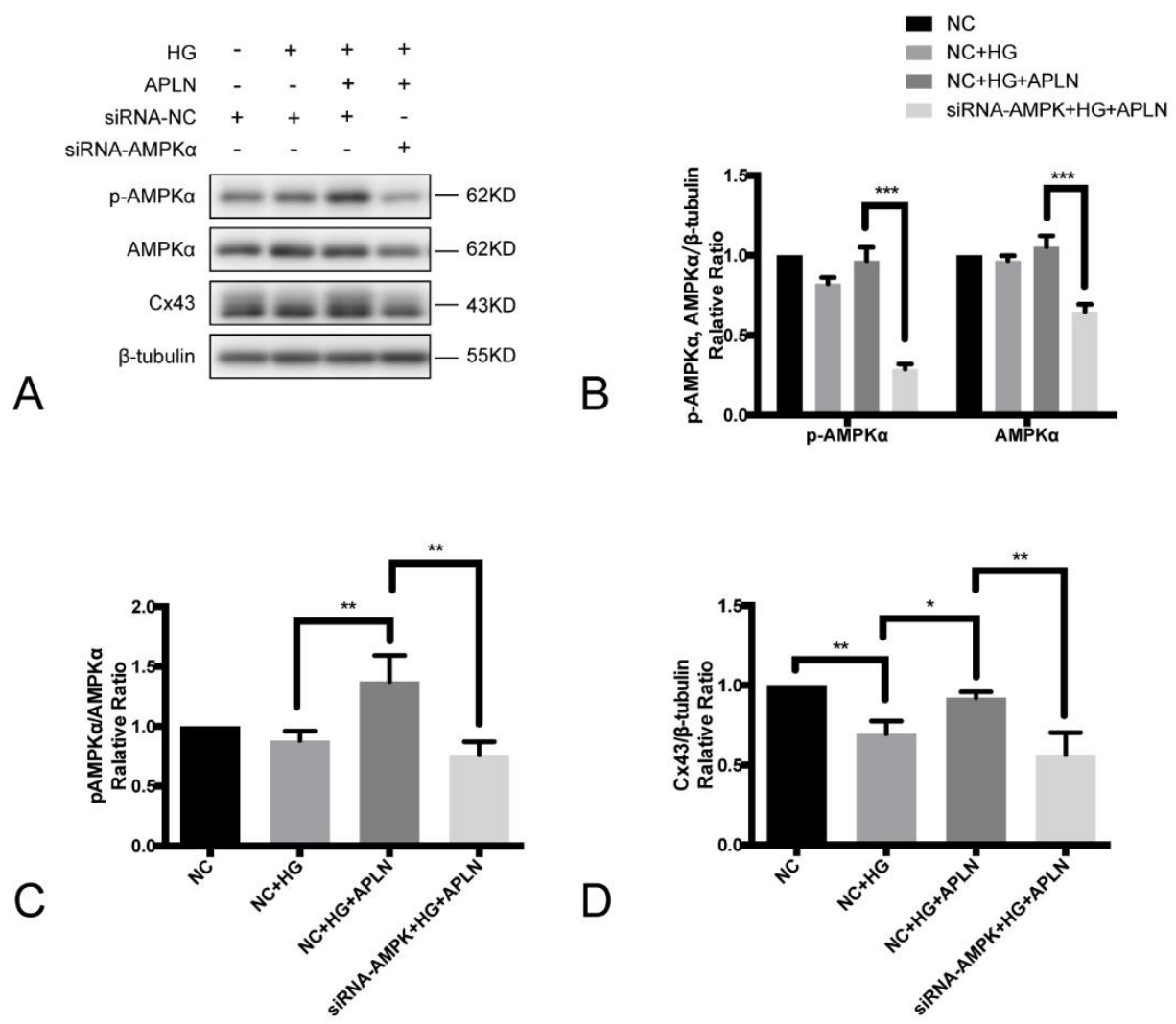

Figure 5. AMPKa suppression inhibited Cx43 expression up-regulated by apelin-13. (A) Western blotting was examined to reveal the expression of p-AMPK $\alpha, \mathrm{AMPK} \alpha$ and $\mathrm{Cx} 43$ in cells transfected with siRNA-NC and siRNA-AMPK $\alpha 1 / \alpha 2$. During the transfection of siRNA, cells were cultured under normal glucose. After $48 \mathrm{~h}$, cells were cultured in high glucose conditions for $72 \mathrm{~h}$ with/without apelin $(100 \mathrm{nM}, 48 \mathrm{~h})$. (B) siRNAAMPK $\alpha 1 / \alpha 2$ significantly suppressed the expression of $\mathrm{p}-\mathrm{AMPK} \alpha$ and AMPK $\alpha$ compared with negative control siRNA. (C) Compared with the high glucose group, apelin-13 addition could increase the p-AMPK $\alpha / A M P K \alpha$ ratio in cells transfected with siRNA-NC, while siRNA-AMPK $\alpha 1 / \alpha 2$ reduced the $\mathrm{p}$-AMPK $\alpha / \mathrm{AMPK} \alpha$ ratio. (D) In cells transfected with negative control siRNA, Cx43 expression was reduced under high glucose and retained with apelin-13 co-treatment. The effect of apelin-13 on Cx43 was abolished by siRNA-AMPK $\alpha 1 / \alpha 2$. SiRNANC: negative control siRNA; $* P<0.05$, $* * P<0.01$, $* * * P<0.001$. Data are the mean \pm SD.

\section{High glucose inhibited AMPK activity while apelin-13 activated AMPK in NRCMs.}

To investigate the alterations of AMPK activity by stimulation, cells were treated with high glucose $(30 \mathrm{mM})$ or apelin-13 (100 nM) for $7.5 \mathrm{~min}, 15 \mathrm{~min}, 30 \mathrm{~min}$ and 60 min. Western blotting analysis showed that the ratio of $\mathrm{p}-$ AMPK $\alpha$ to AMPK $\alpha$ protein significantly decreased under high glucose stimulation and increased under apelin-13 interference (Fig. 3A and 3B). AMPK $\alpha$ activation by apelin-13 remained in cells pre-treated with high glucose (30 mM) for $24 \mathrm{~h}$ (Fig. 3C).

\section{AICAR upregulated pAMPKa and Cx43 expression in NRCMs.}

Since we found that apelin-13 could increase AMPK $\alpha$, AICAR (an AMPK activator) was used as positive control. NRCMs were treated with AICAR $(0.5 \mathrm{mM})$ for 48h to observe the AMPK activity and Cx43 expression. Mannitol here was an osmotic control. The Cx43 expression and pAMPK/AMPK $\alpha$ ratio between control and mannitol group was similar. High glucose $(30 \mathrm{mM}$, 72h) apparently reduced $\mathrm{Cx} 43$ and pAMPK $\alpha$ expression while the addition of apelin $(100 \mathrm{nM}, 48 \mathrm{~h})$ relieved the decline. Compared with the control group, AICAR significantly stimulated AMPK $\alpha$ phosphorylation and enhanced $\mathrm{Cx} 43$ expression (Fig. 5). These data further implied the correlation between AMPK activity and $\mathrm{Cx} 43$ expression. 


\section{Silenced AMPKa expression inhibited Cx43 expression and abolished the protective effect of apelin-13.}

To determine the role of AMPK in $\mathrm{Cx} 43$ expression, RNA interference technology was employed to suppress AMPK $\alpha$ expression. Western blotting was applied to show the expression of p-AMPK $\alpha, A M P K \alpha$ and $C x 43$ in cells transfected with the negative control siRNA and siRNA-AMPK $\alpha 1 / \alpha 2$ (Fig. 5A). In cells co-treated with high glucose and apelin-13, siRNA-AMPK notably repressed the expression of AMPK and phospho-AMPK compared with the negative control siRNA (Fig. 5B). siRNA-AMPK $\alpha 1$ and siRNA-AMPK $\alpha 2$ reduced the ratio of $\mathrm{p}$-AMPK $\alpha$ to AMPK $\alpha$ protein and abolished the $\mathrm{Cx} 43$ abundance induced by apelin-13 (Fig. 5C and 5D). All these observations indicated that the AMPK signaling pathway might be partially involved in the protective effect of apelin- 13 on $\mathrm{Cx} 43$ protein expression reduced by high glucose.

\section{DISCUSSION}

A novel feature of our study is that we identified the protective effect of apelin-13 on gap junctions in cultured cardiomyocytes. Previous studies $[12,13]$ have reported that the expression of $\mathrm{Cx} 43$ was reduced by high glucose in neonatal rat cardiomyocytes. In this work, we found that although either high glucose or apelin-13 did not affect the level of $\mathrm{Cx} 43$ mRNA, apelin-13 attenuated the high glucose-induced $\mathrm{Cx} 43$ protein down-regulation and functional impairment of GJIC. The AMPK signaling pathway might be implicated in this progress. This conclusion was supported by the findings that high glucose inhibited AMPK $\alpha$, while apelin-13 activated the phosphorylation of AMPK $\alpha$, and silenced AMPK $\alpha$ expression abolished the protective effect of apelin-13.

The diminished expression and heterogeneous distribution of $\mathrm{Cx} 43$ impaired the function of gap junctions, resulting in conduction defects in the heart [36, 37]. In streptozotocin (STZ)-induced diabetic rat models, the expression of $\mathrm{Cx} 43$ fluctuated in several studies with differences in STZ concentrations and durations of STZ injections. High glucose contributed to the decrease of Cx43 in a 3-week STZ-induced diabetic rat model [38, 39]. However, the increase of $\mathrm{Cx} 43$ in a 12-week diabetic rat model [7] may have been resulted from accumulated advanced glycation end products (AGEs) from long-term complications of diabetes mellitus [40]. Additionally, our previous study detected $\mathrm{Cx} 43$ up-regulation and lateralization in AGE-infused rats [41]. Considering the complexity of animal models, NRCMs under high glucose (30 $\mathrm{mM}$, mimic of hyperglycemia) were utilized to investigate the effect of apelin on Cx43 expression in the present study.
Apelin, a small peptide, plays important roles in energy metabolism, regulation of cardiac function and improvement of insulin sensitivity [18]. It has also been demonstrated to induce a positive inotropic response in mouse hearts [19-21]. Cx43 protein expression is increased in mouse and human embryonic stem cells treated with apelin [28]. Our data were consistent with the effect of apelin on $\mathrm{Cx} 43$ up-regulation, and the dye transfer assay indicated that apelin was capable of improving gap junctional communication in cardiomyocytes under high glucose. Since the Cx43 transcript level did not vary with the addition of either high glucose or apelin, we hypothesized that apelin amended $\mathrm{Cx} 43$ protein expression by post-transcriptional regulation, protein translation or protein degradation.

Apart from its key role in regulating energy metabolism, AMPK is also a fundamental regulator of cellular proteostasis [42]. AMPK is composed of a catalytic subunit ( $\alpha 1$ and $\alpha 2)$, a scaffolding subunit ( $\beta 1$ and $\beta 2)$ and a regulatory subunit $(\gamma 1, \gamma 2)$ in cardiac tissue. The $\alpha 1$ - and $\alpha 2$-subunits are encoded by PRKAA1 and PRKAA2, respectively, and threonine 174 (Thr174) on the $\alpha 1$-subunit and Thr172 on the $\alpha 2$-subunit regulate AMPK activation [30, 43]. In addition, AMPK has been identified as a major downstream signaling molecule of Apelin [22]. Previous studies demonstrated that AMPK activated mitochondrial $\mathrm{K}_{\mathrm{ATP}}$ channels, thus increasing $\mathrm{Cx} 43$ expression, improving its distribution and attenuating the arrhythmogenic response to programmed electrical stimulation [44]. And Cx43 was found to be reduced in LKB1 (a protein kinase that activates AMPK) deletion mice.

In accordance with the activated AMPK signaling pathway in type II diabetic mice treated with apelin-13 $[45,46]$, we also found that apelin-13 stimulated AMPKa phosphorylation in NRCMs. In this case, we hypothesized that the protective effect of apelin on $\mathrm{Cx} 43$ expression might be mediated, at least in part, via the AMPK pathway. In our study, high glucose inhibited the phosphorylation of AMPK $\alpha$ in a time-dependent manner while apelin activated AMPK $\alpha$ in NRCMs. The impact of apelin on AMPK $\alpha$ still existed in myocardiocytes preincubated with high glucose for $24 \mathrm{~h}$. The widely-used inhibitor of AMPK, dorsomorphin (also called Compound C), is a selective inhibitor of bone morphogenetic protein (BMP) signaling [47]. BMP4, BMP7 and BMP15 significantly decreased $\mathrm{Cx} 43$ expression in human granulosa cells $[48,49]$. Considering that dorsomorphin could increase $\mathrm{Cx} 43$ expression by inhibiting BMP signaling, it was not an applicable AMPK inhibitor in this study. Our study detected that siRNA-AMPK $\alpha 1$ and siRNA-AMPK $\alpha 2$ could suppress AMPK $\alpha$ expression and abrogate the up-regulation of $\mathrm{Cx} 43$ expression by apelin. 
In consistent with our findings, Guo, et al. observed that expression of $\mathrm{Cx} 43$ and p-AMPK was diminished in diabetic nephropathy specimens and cultured glomerular mesangial cells (GMCs) under high glucose conditions. In cultured GMCs, silenced AMPK expression by siRNA suppressed Cx43 expression while AMPK activation with metformin alleviated the down-regulation of $\mathrm{Cx} 43$ induced by high glucose [34]. However, recent data from Alesutan, et al. suggested that AMPK $\alpha 1$ stimulated ubiquitination of $\mathrm{Cx} 43$, thereby leading to decreased $\mathrm{Cx} 43$ protein abundance in transverse aortic constriction (TAC)-treated mouse cardiac tissue [29]. Possible reasons for the controversial outcomes might be from the different animal models. The fundamental level of AMPK activity varies according to the models of diseases. As previously mentioned, AMPK was stimulated in mice exposured to overload pressure, whereas AMPK activity was inhibited in high glucose-treated GMCs and $\mathrm{H} 9 \mathrm{C} 2$ cardiac myoblast cells [50]. The action of AMPK, whether beneficial or detrimental, remains uncertain as well. We presumed that it was determined by the maintenance of efficient cellular homeostasis.

In summary, this study shed new light on the potential role of apelin in protecting the cardiac gap junctions from remodeling under hyperglycemia. Apelin attenuated high glucose-triggered $\mathrm{Cx} 43$ reduction and improved the function of gap junctions partly through the AMPK pathway. This suggests that apelin-13 might be a novel therapeutic strategy, via regulation of AMPK activity, for arrhythmias in diabetic cardiopathy. Further studies are needed to determine the downstream molecules of AMPK involved in Cx43 alteration and the impact of apelin on gap junctions in animal models.

\section{Acknowledgements}

We thank the Biomedical Research Center at Sir Run Run Shaw Hospital, Zhejiang University School of Medicine for the use of instruments and equipments. This work was supported by the National Natural Science Foundation of China (Project no. 81570246 and 81500212), the Medical and Health Science Program of Zhejiang Province (Project no. 201477370), and the Natural Science Foundation of Zhejiang Province (Project no. LY16H020007). There was no conflict of interests in this work.

\section{References}

[1] Nygren A, Olson ML, Chen KY, Emmett T, Kargacin G, Shimoni Y (2007). Propagation of the cardiac impulse in the diabetic rat heart: reduced conduction reserve. J Physiol, 580: 543-560.
[2] Okruhlicova L, Tribulova N, Misejkova M, Kucka M, Stetka R, Slezak J, et al. (2002). Gap junction remodelling is involved in the susceptibility of diabetic rats to hypokalemia-induced ventricular fibrillation. Acta Histochem, 104: 387-391.

[3] Alexandra F. Bruce SR, Emmanuel Dupont, and Nicholas J. Severs (2008). Gap junction remodelling in human heart failure is associated with increased interaction of connexin43 with ZO-1. Cardiovasc Res, 77: 757-765.

[4] Matsuyama D, Kawahara K (2009). Proliferation of neonatal cardiomyocytes by connexin 43 knockdown via synergistic inactivation of p38 MAPK and increased expression of FGF1. Basic Res Cardiol, 104: 631-642.

[5] Hesketh GG, Shah MH, Halperin VL, Cooke CA, Akar FG, Yen TE, et al. (2010). Ultrastructure and regulation of lateralized connexin43 in the failing heart. Circ Res, 106: 1153-1163.

[6] Su V, Lau AF (2014). Connexins: mechanisms regulating protein levels and intercellular communication. FEBS Lett, 588: 1212-1220.

[7] Howarth FC, Chandler NJ, Kharche S, Tellez JO, Greener ID, Yamanushi TT, et al. (2008). Effects of streptozotocin-induced diabetes on connexin 43 mRNA and protein expression in ventricular muscle. Mol Cell Biochem, 319: 105-114.

[8] Shimoni Y, Emmett T, Schmidt R, Nygren A, Kargacin G (2009). Sex-dependent impairment of cardiac action potential conduction in type 1 diabetic rats. Am J Physiol-Heart C, 296: H1442-1450.

[9] Duffy HS (2012). The molecular mechanisms of gap junction remodeling. Heart rhythm, 9: 1331-1334.

[10] Fontes MS, van Veen TA, de Bakker JM, van Rijen HV (2012). Functional consequences of abnormal Cx43 expression in the heart. Biochim Biophys Acta, 1818: 2020-2029.

[11] Das Evcimen N, King GL (2007). The role of protein kinase $\mathrm{C}$ activation and the vascular complications of diabetes. Pharmacol Res, 55: 498-510.

[12] Yu L, Zhao Y, Fan Y, Wang M, Xu S, Fu G (2010). Epigallocatechin-3 gallate, a green tea catechin, attenuated the downregulation of the cardiac gap junction induced by high glucose in neonatal rat cardiomyocytes. Cell Physiol Biochem, 26: 403-412.

[13] Yu L, Yu H, Li X, Jin C, Zhao Y, Xu S, et al. (2016). $\mathrm{P} 38 \mathrm{MAPK} / \mathrm{miR}-1$ are involved in the protective effect of EGCG in high glucose-induced $\mathrm{Cx} 43$ downregulation in neonatal rat cardiomyocytes. Cell Biol Int, 40: 934942.

[14] Tatemoto K, Hosoya M, Habata Y, Fujii R, Kakegawa T, Zou MX, et al. (1998). Isolation and characterization of a novel endogenous peptide ligand for the human APJ receptor. Biochem Biophys Res Commun, 251: 471476.

[15] Zeng X-XI, Wilm TP, Sepich DS, Solnica-Krezel L (2007). Apelin and Its Receptor Control Heart Field Formation during Zebrafish Gastrulation. Dev Cell, 12: 391-402.

[16] Bertrand C, Valet P, Castan-Laurell I (2015). Apelin and energy metabolism. Front Physiol, 6: 115. 
[17] Chaves-Almagro C, Castan-Laurell I, Dray C, Knauf C, Valet P, Masri B (2015). Apelin receptors: From signaling to antidiabetic strategy. Eur J Pharmacol, 763: 149-159.

[18] Castan-Laurell I, Dray C, Knauf C, Kunduzova O, Valet $P$ (2012). Apelin, a promising target for type 2 diabetes treatment? Trends Endocrinol Metab, 23: 234-241.

[19] Szokodi I (2002). Apelin, the Novel Endogenous Ligand of the Orphan Receptor APJ, Regulates Cardiac Contractility. Circ Res, 91: 434-440.

[20] Ashley EA, Powers J, Chen M, Kundu R, Finsterbach T, Caffarelli A, et al. (2005). The endogenous peptide apelin potently improves cardiac contractility and reduces cardiac loading in vivo. Cardiovasc Res, 65: 7382.

[21] Kuba K, Zhang L, Imai Y, Arab S, Chen M, Maekawa Y, et al. (2007). Impaired heart contractility in Apelin gene-deficient mice associated with aging and pressure overload. Circ Res, 101: e32-42.

[22] Simpkin JC, Yellon DM, Davidson SM, Lim SY, Wynne AM, Smith CC (2007). Apelin-13 and apelin-36 exhibit direct cardioprotective activity against ischemiareperfusion injury. Basic Res Cardiol, 102: 518-528.

[23] Rastaldo R, Cappello S, Folino A, Berta GN, Sprio AE, Losano G, et al. (2011). Apelin-13 limits infarct size and improves cardiac postischemic mechanical recovery only if given after ischemia. Am J Physiol Heart Circ Physiol, 300: H2308-2315.

[24] $\mathrm{Hu}$ H, He L, Li L, Chen L (2016). Apelin/APJ system as a therapeutic target in diabetes and its complications. Mol Genet Metab, 119: 20-27.

[25] Ellinor PT, Low AF, Macrae CA (2006). Reduced apelin levels in lone atrial fibrillation. Eur Heart J, 27: 222-226.

[26] Falcone C, Buzzi MP, D'Angelo A, Schirinzi S, Falcone R, Rordorf R, et al. (2010). Apelin plasma levels predict arrhythmia recurrence in patients with persistent atrial fibrillation. Int J Immunopathol Pharmacol, 23: 917925.

[27] Kallergis EM, Manios EG, Kanoupakis EM, Mavrakis HE, Goudis CA, Maliaraki NE, et al. (2010). Effect of sinus rhythm restoration after electrical cardioversion on apelin and brain natriuretic Peptide prohormone levels in patients with persistent atrial fibrillation. Am J Cardiol, 105: 90-94.

[28] Wang IN, Wang X, Ge X, Anderson J, Ho M, Ashley E, et al. (2012). Apelin enhances directed cardiac differentiation of mouse and human embryonic stem cells. PloS one, 7: e38328.

[29] Alesutan I, Voelkl J, Stockigt F, Mia S, Feger M, Primessnig U, et al. (2015). AMP-activated protein kinase alpha1 regulates cardiac gap junction protein connexin 43 and electrical remodeling following pressure overload. Cell Physiol Biochem, 35: 406-418.

[30] Bairwa SC, Parajuli N, Dyck JRB (2016). The role of AMPK in cardiomyocyte health and survival. Biochim Biophys Acta, 1862: 2199-2210.

[31] Zhang X, Yao J, Gao K, Chi Y, Mitsui T, Ihara T, et al. (2016). AMPK Suppresses Connexin43 Expression in the Bladder and Ameliorates Voiding Dysfunction in
Cyclophosphamide-induced Mouse Cystitis. Sci Rep, 6: 19708.

[32] Harada M, Nattel SN, Nattel S (2012). AMP-activated protein kinase: potential role in cardiac electrophysiology and arrhythmias. Circ Arrhythm Electrophysiol, 5: 860-867.

[33] Chen F, Zhao WT, Chen FX, Fu GS, Mou Y, Hu SJ (2014). High glucose promotes gap junctional communication in cultured neonatal cardiac fibroblasts via AMPK activation. Mol Biol (Mosk), 48: 599-606.

[34] Guo YN, Wang JC, Cai GY, Hu X, Cui SY, Lv Y, et al. (2014). AMPK-mediated downregulation of connexin 43 and premature senescence of mesangial cells under highglucose conditions. Exp Gerontol, 51: 71-81.

[35] Babica P, Sovadinova I, Upham BL (2016). Scrape Loading/Dye Transfer Assay. Methods Mol Biol, 1437: 133-144.

[36] Akar FG, Nass RD, Hahn S, Cingolani E, Shah M, Hesketh GG, et al. (2007). Dynamic changes in conduction velocity and gap junction properties during development of pacing-induced heart failure. Am J Physiol Heart Circ Physiol, 293: H1223-1230.

[37] Chkourko HS, Guerrero-Serna G, Lin X, Darwish N, Pohlmann JR, Cook KE, et al. (2012). Remodeling of mechanical junctions and of microtubule-associated proteins accompany cardiac connexin43 lateralization. Heart rhythm, 9: 1133-1140 e1136.

[38] Lin H, Ogawa K, Imanaga I, Tribulova N (2006). Remodeling of connexin 43 in the diabetic rat heart. Mol Cell Biochem, 290: 69-78.

[39] Stilli D, Lagrasta C, Berni R, Bocchi L, Savi M, Delucchi F, et al. (2007). Preservation of ventricular performance at early stages of diabetic cardiomyopathy involves changes in myocyte size, number and intercellular coupling. Basic Res Cardiol, 102: 488-499.

[40] Hou X, Hu Z, Xu H, Xu J, Zhang S, Zhong Y, et al. (2014). Advanced glycation endproducts trigger autophagy in cadiomyocyte via RAGE/PI3K/AKT/mTOR pathway. Cardiovasc Diabetol, 13: 78.

[41] Yu L, Zhao Y, Xu S, Ding F, Jin C, Fu G, et al. (2013). Advanced Glycation End Product (AGE)-AGE Receptor (RAGE) System Upregulated Connexin43 Expression in Rat Cardiomyocytes via PKC and Erk MAPK Pathways. Int J Mol Sci, 14: 2242-2257.

[42] Salminen A, Kaarniranta K, Kauppinen A (2016). Agerelated changes in AMPK activation: Role for AMPK phosphatases and inhibitory phosphorylation by upstream signaling pathways. Ageing Res Rev, 28: 1526.

[43] Cameron KO, Kurumbail RG (2016). Recent progress in the identification of adenosine monophosphateactivated protein kinase (AMPK) activators. Bioorg Med Chem Lett, 26: 5139-5148.

[44] Qiu J, Zhou S, Liu Q (2016). Phosphorylated AMPactivated protein kinase slows down the atrial fibrillation progression by activating Connexin43. Int $\mathbf{J}$ Cardiol, 208: 56-57.

[45] Dray C, Knauf C, Daviaud D, Waget A, Boucher J, Buleon M, et al. (2008). Apelin stimulates glucose 
utilization in normal and obese insulin-resistant mice. Cell Metab, 8: 437-445.

[46] Chen H, Li J, Jiao L, Petersen RB, Li J, Peng A, et al. (2014). Apelin inhibits the development of diabetic nephropathy by regulating histone acetylation in Akita mouse. J Physiol, 592: 505-521.

[47] Hao J, Daleo MA, Murphy CK, Yu PB, Ho JN, Hu J, et al. (2008). Dorsomorphin, a selective small molecule inhibitor of BMP signaling, promotes cardiomyogenesis in embryonic stem cells. PLoS One, 3: e2904.

[48] Chang HM, Cheng JC, Leung PC (2013). Theca-derived BMP4 and BMP7 down-regulate connexin43 expression and decrease gap junction intercellular communication activity in immortalized human granulosa cells. J Clin Endocrinol Metab, 98: E437-445.

[49] Chang HM, Cheng JC, Taylor E, Leung PC (2014). Oocyte-derived BMP15 but not GDF9 down-regulates connexin43 expression and decreases gap junction intercellular communication activity in immortalized human granulosa cells. Mol Hum Reprod, 20: 373-383.

[50] He C, Zhu H, Li H, Zou MH, Xie Z (2013). Dissociation of Bcl-2-Beclin1 complex by activated AMPK enhances cardiac autophagy and protects against cardiomyocyte apoptosis in diabetes. Diabetes, 62: 1270-1281. 